\title{
Capacity of a simultaneous quantum secure direct communication scheme between the central party and other $M$ parties
}

\author{
Ting Gao ${ }^{1,2}$, Feng-Li Yan ${ }^{2,3}$, Zhi-Xi Wang ${ }^{4}$, You-Cheng Li ${ }^{2,3}$ \\ 1 College of Mathematics and Information Science, \\ Hebei Normal University, Shijiazhuang 050016, China \\ 2 CCAST (World Laboratory), \\ P.O. Box 8730, Beijing 100080, China \\ 3 College of Physics and Information Engineering, \\ Hebei Normal University, Shijiazhuang 050016, China \\ 4 Department of Mathematics, \\ Capital Normal University, Beijing 100037, China
}

(Dated: August 6, 2018)

\begin{abstract}
We analyze the capacity of a simultaneous quantum secure direct communication scheme between the central party and other $M$ parties via $M+1$-particle GHZ states and swapping quantum entanglement. It is shown that the encoding scheme should be secret if other $M$ parties wants to transmit $M+1$ bit classical messages to the center party secretly. However when the encoding scheme is announced publicly, we prove that the capacity of the scheme in transmitting the secret messages is 2 bits, no matter how big $M$ is.

PACS numbers: 03.67.Hk
\end{abstract}

Quantum cryptography or quantum key distribution (QKD) utilizing the features of quantum mechanics, such as uncertainty principle, quantum correlations and nonlocality, is one of the most promising applications of quantum information science and is considered to be the safest system to enable the provable secure distribution of private information among the authorized parties. It has attracted widespread attention since the seminal work on QKD by Bennett and Brassard (BB84) [1]. As shown in this protocol, two remote authorized users, Alice and Bob, can establish a shared secret key with nonorthogonal polarized single photons. The quantum non-cloning theorem 2] and the tradeoff between information gain and disturbance [3] guarantee strongly the unconditionally secure of the BB84 protocol [4]. In virtue of correlation of an Einstein-Podolsky-Rosen (EPR) pair, the maximally entangled two-particle state, Ekert proposed a QKD protocol, in which the Bell inequality is used for detecting the eavesdropper Eve 5]. Since then there have been various theoretical proposals [6, 7, 8, 9, 10, 11], which have been summed up in a review paper [12].

Recently Shimizu and Imoto [13, 14] and Beige et al [15] came up with a novel quantum secure direct communication (QSDC) schemes, which allows information to be transmitted in a deterministic secure way. Boström and Felbinger presented a QSDC protocol called the "ping-pong protocol" based on entanglement and twoway communication [16]. Wójcik investigated the security of the ping-pong protocol in a noisy quantum channel [17. The ping-pong protocol was modified by Cai and Li with single photons 18]. Deng et al introduced two QSDC schemes, one using EPR pair block 19] and the other based on polarized single photons 20]. A QSDC scheme with quantum superdense coding [21] was designed by Wang et al. Some ideas in entanglement swap- ping has been exploited for QSDC by Man, Zhang and Li 22]. Zhu, Xia, Fan and Zhang put forward a QSDC protocol based on secret transmitting order of particles 23. Cao and Song proposed a QSDC protocol with W state [24]. In virtue of quantum teleportation and entanglement swapping we suggested several QSDC schemes 25, 26, 27, 28, 29, 30, 31]. Recently, the QSDC has been extended to multiparty quantum secret report by Deng et al 32 .

In Ref. 31] we presented a simultaneous quantum secure direct communication scheme between the central party and other $M$ parties, which utilizes shared $M+1$ particle Greenberger-Horne-Zeilinger (GHZ) states and entanglement swapping between communicating parties. For the sake of simplicity in [31], we mainly paid our attention to the protocol in the case of $M=3$, which is briefly described as follows. Suppose that a lot of fourparty GHZ states (GHZ quadruplets)

$$
|G H Z\rangle=\frac{1}{\sqrt{2}}(|0000\rangle+|1111\rangle)
$$

are shared by the three spatially separated senders, Alice, Bob and Charlie and a remote center party (receiver) Diana. After ensuring the safety of the quantum channel, Alice, Bob and Charlie encode secret classical bits by applying predetermined unitary operations (encoding scheme) on GHZ quartets. Then Alice, Bob, Charlie and Diana make Bell state measurements on each GHZ quartet pair composed of one GHZ state encoded by the senders and another original GHZ quartet. Based on Alice, Bob and Charlie's Bell state measurement results announced publicly and Diana's outcome which is kept in secret, Diana can infer the secret messages of the senders.

In [31] we did not state clearly whether the encoding scheme is secret or not. However, it is the encoding 
scheme that determines the capacity of the communication scheme. In present paper we will investigate this problem and show that the encoding scheme should be secret if other $M$ parties wants to transmit $M+1$ bit classical secret messages to the center party; but when the encoding scheme is announced publicly, the capacity of the scheme in transmitting the secret messages is 2 bits, no matter how big $M$ is.

Let us analyze the capacity of the scheme in transmitting the secret message when the encoding scheme is announced publicly. Without loss of generality, we consider the case of $M=3$.

The encoding scheme used in the protocol 31] is that Alice applies the unitary operations

$$
\begin{gathered}
\sigma_{00}^{A}=I=|0\rangle\langle 0|+| 1\rangle\left\langle 1\left|, \quad \sigma_{01}^{A}=\sigma_{x}=\right| 0\right\rangle\langle 1|+| 1\rangle\langle 0|, \\
\sigma_{10}^{A}=\mathrm{i} \sigma_{y}=|0\rangle\langle 1|-| 1\rangle\left\langle 0\left|, \quad \sigma_{11}^{A}=\sigma_{z}=\right| 0\right\rangle\langle 0|-| 1\rangle\langle 1|
\end{gathered}
$$

on GHZ quadruplets, and encode two bits classical information as

$$
\sigma_{00}^{A} \rightarrow 00, \sigma_{01}^{A} \rightarrow 01, \sigma_{10}^{A} \rightarrow 10, \sigma_{11}^{A} \rightarrow 11 .
$$

Meanwhile, Diana and Bob, and Diana and Charlie agree on that Bob and Charlie can only apply unitary operations

$$
\sigma_{0}^{X}=I=|0\rangle\langle 0|+| 1\rangle\left\langle 1\left|, \sigma_{1}^{X}=\sigma_{x}=\right| 0\right\rangle\langle 1|+| 1\rangle\langle 0|
$$

to encode one bit classical information as following

$$
\sigma_{0}^{X} \rightarrow 0, \sigma_{1}^{X} \rightarrow 1
$$

respectively. Here X can be either B or C.

Suppose that Alice, Bob, Charlie, and Diana initially share GHZ quadruplets $|G H Z\rangle_{1234}$ and $|G H Z\rangle_{5678}$, where particles 1 and 5,2 and 6,3 and 7 , and 4 and 8 belong to Alice, Bob, Charlie and Diana respectively. After senders encode their secret messages by applying predetermined unitary operators on $|G H Z\rangle_{1234}$, $|G H Z\rangle_{1234}$ becomes $\sigma_{i j}^{A} \otimes \sigma_{l}^{B} \otimes \sigma_{m}^{C}|G H Z\rangle_{1234}$, where $i, j, l, m \in\{0,1\}$.
It is easy to prove that

$$
\begin{aligned}
& \sigma_{i j}^{A} \otimes \sigma_{l}^{B} \otimes \sigma_{m}^{C}|G H Z\rangle_{1234} \otimes|G H Z\rangle_{5678} \\
= & \frac{1}{4}\left[\sigma_{i j}^{A} \Phi_{15}^{+} \otimes \sigma_{l}^{B} \Phi_{26}^{+} \otimes \sigma_{m}^{C} \Phi_{37}^{+} \otimes \Phi_{48}^{+}\right. \\
& +\sigma_{i j}^{A} \Phi_{15}^{+} \otimes \sigma_{l}^{B} \Phi_{26}^{+} \otimes \sigma_{m}^{C} \Phi_{37}^{-} \otimes \Phi_{48}^{-} \\
& +\sigma_{i j}^{A} \Phi_{15}^{+} \otimes \sigma_{l}^{B} \Phi_{26}^{-} \otimes \sigma_{m}^{C} \Phi_{37}^{+} \otimes \Phi_{48}^{-} \\
& +\sigma_{i j}^{A} \Phi_{15}^{+} \otimes \sigma_{l}^{B} \Phi_{26}^{-} \otimes \sigma_{m}^{C} \Phi_{37}^{-} \otimes \Phi_{48}^{+} \\
& +\sigma_{i j}^{A} \Phi_{15}^{-} \otimes \sigma_{l}^{B} \Phi_{26}^{+} \otimes \sigma_{m}^{C} \Phi_{37}^{+} \otimes \Phi_{48}^{-} \\
& +\sigma_{i j}^{A} \Phi_{15}^{-} \otimes \sigma_{l}^{B} \Phi_{26}^{+} \otimes \sigma_{m}^{C} \Phi_{37}^{-} \otimes \Phi_{48}^{+} \\
& +\sigma_{i j}^{A} \Phi_{15}^{-} \otimes \sigma_{l}^{B} \Phi_{26}^{-} \otimes \sigma_{m}^{C} \Phi_{37}^{+} \otimes \Phi_{48}^{+} \\
& +\sigma_{i j}^{A} \Phi_{15}^{-} \otimes \sigma_{l}^{B} \Phi_{26}^{-} \otimes \sigma_{m}^{C} \Phi_{37}^{-} \otimes \Phi_{48}^{-} \\
& +\sigma_{i j}^{A} \Psi_{15}^{+} \otimes \sigma_{l}^{B} \Psi_{26}^{+} \otimes \sigma_{m}^{C} \Psi_{37}^{+} \otimes \Psi_{48}^{+} \\
& +\sigma_{i j}^{A} \Psi_{15}^{+} \otimes \sigma_{l}^{B} \Psi_{26}^{+} \otimes \sigma_{m}^{C} \Psi_{37}^{-} \otimes \Psi_{48}^{-} \\
& +\sigma_{i j}^{A} \Psi_{15}^{+} \otimes \sigma_{l}^{B} \Psi_{26}^{-} \otimes \sigma_{m}^{C} \Psi_{37}^{+} \otimes \Psi_{48}^{-} \\
& +\sigma_{i j}^{A} \Psi_{15}^{+} \otimes \sigma_{l}^{B} \Psi_{26}^{-} \otimes \sigma_{m}^{C} \Psi_{37}^{-} \otimes \Psi_{48}^{+} \\
& +\sigma_{i j}^{A} \Psi_{15}^{-} \otimes \sigma_{l}^{B} \Psi_{26}^{+} \otimes \sigma_{m}^{C} \Psi_{37}^{+} \otimes \Psi_{48}^{-} \\
& +\sigma_{i j}^{A} \Psi_{15}^{-} \otimes \sigma_{l}^{B} \Psi_{26}^{+} \otimes \sigma_{m}^{C} \Psi_{37}^{-} \otimes \Psi_{48}^{+} \\
& +\sigma_{i j}^{A} \Psi_{15}^{-} \otimes \sigma_{l}^{B} \Psi_{26}^{-} \otimes \sigma_{m}^{C} \Psi_{37}^{+} \otimes \Psi_{48}^{+} \\
& \left.+\sigma_{i j}^{A} \Psi_{15}^{-} \otimes \sigma_{l}^{B} \Psi_{26}^{-} \otimes \sigma_{m}^{C} \Psi_{37}^{-} \otimes \Psi_{48}^{-}\right] .
\end{aligned}
$$

Here

$$
\Phi^{ \pm} \equiv \frac{1}{\sqrt{2}}(|00\rangle \pm|11\rangle), \Psi^{ \pm} \equiv \frac{1}{\sqrt{2}}(|01\rangle \pm|10\rangle)
$$

are four Bell states (EPR pairs). By virtue of Eq.(6), it is not difficult to observe that only four unitary operators $\sigma_{i j}^{A} \otimes \sigma_{l}^{B} \otimes \sigma_{m}^{C}$ can correspond to a fixed outcome of Alice, Bob and Charlie's Bell state measurements. For example, if Alice obtains measurement result $\Psi_{15}^{+}$, Bob $\Phi_{26}^{+}$, and Charlie $\Psi_{37}^{+}$, we can see that only $\sigma_{00}^{A} \otimes \sigma_{1}^{B} \otimes \sigma_{0}^{C}$, $\sigma_{01}^{A} \otimes \sigma_{0}^{B} \otimes \sigma_{1}^{C}, \sigma_{10}^{A} \otimes \sigma_{0}^{B} \otimes \sigma_{1}^{C}, \sigma_{11}^{A} \otimes \sigma_{1}^{B} \otimes \sigma_{0}^{C}$ can make $|G H Z\rangle_{1234} \otimes|G H Z\rangle_{5678}$ to cause this measurement result $\Psi_{15}^{+}, \Phi_{26}^{+}$, and $\Psi_{37}^{+}$. So the capacity of the scheme in transmitting the secret messages is 2 bits when the encoding scheme is announced publicly. For the case $M>3$ one can obtain the same result. That is, when the encoding scheme is announced publicly, the capacity of the scheme is 2 bits, no matter how big $M$ is.

Now let us consider this problem from another point of view. Obviously only based on Diana's Bell state measurement result, can Diana infer the senders' secret message, as the senders outcomes of the Bell state are announced publicly. However as a matter of fact, Diana can only obtain four different results in a Bell state measurement. Therefore the capacity of the scheme in transmitting the secret messages can not beyond 2 bits.

Fortunately, if the encoding scheme (for example Eqs.(2)-(5)) is kept in secret, the situation would be changed, as there are many encoding schemes. That is, if 
the information on the encoding scheme is not available to the eavesdropper, there is no way for the eavesdropper to find correct classical secret bits with a probability more than $\frac{1}{2^{M+1}}$. Therefore in this case the capacity is also $M+1$ bits. In other words, if the encoding scheme is secret then other $M$ parties can transmit $M+1$ bit classical messages to the center party.

In summary, we have analyzed the capacity of a simultaneous quantum secure direct communication scheme between the central party and other $M$ parties via $M+1$ particle GHZ states and swapping quantum entanglement. It is shown that the encoding scheme should be secret if other $M+1$ parties wants to transmit $M+1$ bit classical messages to the center party secretly. However when the encoding scheme is announced publicly, we prove that the capacity of the scheme in transmitting the secret message is 2 bits, no matter how big $M$ is.

\section{Acknowledgments}

The authors sincerely thank Prof. Long Gui-Lu and Dr. Deng Fu-Guo for many insightful discussions and helpful comments on the manuscript. This work was supported by Hebei Natural Science Foundation of China under Grant Nos: A2004000141 and A2005000140, and Key Natural Science Foundation of Hebei Normal University.
[1] Bennett C H and Brassard G Proc. IEEE Int. Conf. on Computers, Systems and Signal Processing, Bangalore, India, (IEEE, New York, 1984), pp. 175-179

[2] Wootters W K and Zurek W H 1982 Nature 299802

[3] Nielsen M A and Chuang I L 2000 Quantum Computation and Quantum Information (Cambridge: Cambridge University Press)

[4] Shor P W and Preskill J 2000 Phys. Rev. Lett. 85441

[5] Ekert A K 1991 Phys. Rev. Lett. 67661

[6] Bennett C H 1992 Phys. Rev. Lett. 683121

[7] Bennett C H, Brassard G and Mermin N D 1992 Phys. Rev. Lett. $\mathbf{6 8} 557$

[8] Long G L and Liu X S 2002 Phys. Rev. A 65032302

[9] Xue P, Li C F and Guo G C 2002 Phys. Rev. A 65022317

[10] Deng F G and Long G L 2003 Phys. Rev. A 68042315

[11] Deng F G and Long G L 2004 Phys. Rev. A 70012311

[12] Gisin N, Ribordy G, Tittel W and Zbinden H 2002 Rev. Mod. Phys. $\mathbf{7 4} 145$

[13] Shimizu K and Imoto N 1999 Phys. Rev. A 60157

[14] Shimizu K and Imoto N 2000 Phys. Rev. A 62054303

[15] Beige A et al 2002 Acta Phys. Pol. A 101357

[16] Boström K and Felbinger T 2002 Phys. Rev. Lett. 89187902

[17] Wójcik A 2003 Phys. Rev. Lett. 90157901
[18] Cai Q Y and Li B W 2004 Chin. Phys. Lett. 21601

[19] Deng F G, Long G L and Liu X S 2003 Phys. Rev. A 68 042317

[20] Deng F G and Long G L 2004 Phys. Rev. A 69052319

[21] Wang C, Deng F G, Li Y S, Liu X S and Long G L 2005 Phys. Rev. A $\mathbf{7 1} 044305$

[22] Man Z X, Zhang Z J and Li Y 2005 Chin. Phys. Lett. 2218

[23] Zhu A D, Xia Y, Fan Q B and Zhang S 2006 Phys. Rev. A 73 022388

[24] Cao H J and Song H S 2006 Chin. Phys. Lett. 23290

[25] Yan F L and Zhang X Q 2004 Eur. Phys. J. B 4175

[26] Gao T 2004 Z. Naturforsch. 59a 597

[27] Gao T, Yan F L and Wang Z X 2005 Chin. Phys. 14893

[28] Gao T, Yan F L and Wang Z X 2005 Int. J. Mod. Phys. C 16 1293

[29] Gao T, Yan F L and Wang Z X 2004 Il Nuovo Cimento 119B 313

[30] Gao T, Yan F L and Wang Z X 2005 J. Phys. A 385761

[31] Gao T, Yan F L and Wang Z X 2005 Chin. Phys. Lett. 22 2473

[32] Deng F G, Li X H, Li C Y, Zhou P, Liang Y J and Zhou H $\mathrm{Y}$, to be published. 